\title{
A SURVEY ON NATURAL COMPUTING TECHNIQUES
}

\author{
Amit Kumar ${ }^{1}$, Sanjay Singla ${ }^{2}$, Meenakshi Sharma ${ }^{3}$ \& S.N Panda ${ }^{4}$
}

\begin{abstract}
This paper discusses various natural computing techniques including particle swarm optimization, genetic algorithm, moth flame optimization etc. These techniques can be applied in the wireless sensor network to optimize the performance of the network. This paper also discusses the implementation of different natural computing techniques in the wireless sensor network. The paper describes the moth flame optimization algorithm (MFOA) in detail along with the modified MFOA for routing in the wireless sensor network. The algorithm can be used for optimized routing in the wireless sensor network.
\end{abstract}

Keywords: Optimization, Moth, Moth-flame, natural computing, particle swarm optimization

\section{INTRODUCTION}

Natural Computing is a field of research that statements against the specialization of requests in science. It shows up, with its three standard areas of examination, that data from various fields of research are vital for an unrivaled cognizance of life, for the audit and propagation of trademark systems and frames, and for the recommendation of novel enlisting perfect models [1]. Physicists, logical specialists, engineers, analyst, PC scientists, among others, all need to act together or conceivably share musings and data with a particular true objective to make ordinary enrolling useful. Most of the computational approaches regular preparing oversees rely upon exceedingly streamlined variations of the instruments and strategies present in the looking at ordinary marvels. The reasons behind such enhancements and consultations are mind boggling. As an issue of first significance, most separations are vital to make the estimation with a broad number of components tractable. Similarly, it can be productive to feature the inconsequential segments essential to engage some particular parts of a system to be reproduced and to observe some eminent properties. These eminent properties prompt the utilization of the nature enlivened calculations for heuristic pursuit. In this way, nature enlivened systems can be utilized for advancement [2].

Different nature propelled approaches given by various analysts are Genetic Algorithm (GA) [3], Particle Swarm Optimization (PSO) [4], Differential Evolution (DE) [5], Gravitational Search Algorithm (GSA) [6], Gray Wolf Optimizer (GWO) [7], Moth-Flame Optimization Algorithm (MFOA) [1] and so forth. GA shows the conduct of organic development, finishes its enhancement operation by utilizing the hybrid and transformation administrators. Non-overwhelmed Sorting hereditary calculation (NSGA) is a well known multi-target EC calculation which is an adjustment of hereditary calculation. This calculation manufactures the parent populace for the cutting edge by arranging all guardians and posterity into various level of non-ruled arrangements. This calculation likewise protects the decent variety in light of the swarming separation [8][9]. The PSO exhibit the movement of flying creature rush, the speed and position updation wonders of the feathered creature run are utilized for the advancement. Adjusted paired molecule swarm streamlining (MBPSO) is intended to manage untimely meeting of twofold molecule swarm enhancement. It deals with the molecule irregularity utilizing the speed and the similarity between best molecule arrangements [10]. DE advances the issue by refreshing the applicant arrangement in lieu to a given measure of value. The GSA utilizes the gravitational drive essentials for the improvement reason. The MFOA utilizes the moth-fire steering wonder to streamline any issue. The MFOA is portrayed in the following segment. The rest of the paper has been grouped in to four areas. The following area covers the MFOA calculation took after by the segment containing point of interest of dolphin. At that point moth-dolphin calculation and its execution assessment has been done in next two segments. Remote sensor frameworks have wandered into a convincing logbook to assemble data of the earth. Particular little, time tested and abhorrent identifying segments are used to get recognized information [11]. Likewise, due to the headway of remote framework, an enormous number of sensors are started in an extraordinarily selected manner to precisely notice and portrayal the condition of a territory. In this way, remote sensor frameworks are tolerating further applications in different practices, for instance, water watching, battle region surveillance and fire caution. In the interim, many sorts of sensor tradition are considered to make straightforward the limit of sensor frameworks [12]. Nearby every one of the traditions, the power capable data get together tradition is incredibly essential to remain the framework work proposed for the wide time span. When in doubt, information can be amassed by three courses are as: tree-based, bunch based and chain-based [13].

Directing in sensor frameworks is outstandingly trying a direct result of a couple of characteristics that remember them from contemporary correspondence and remote uniquely selected frameworks. Remote sensor frameworks are system less, remote associations are conflicting [14]. The sensor centers are solidly constrained the extent that transmission control, and along

\footnotetext{
${ }^{1}$ Corresponding author, Phd Scholar, Department of Computer Science \& Engineering, PTU University Jalandhar, Punjab India

${ }^{2}$ Department of Computer Science \& Engineering, IET Bhaddal Technical Campus, Ropar, Punjab

${ }^{3}$ University Institute of Computing, Chandigarh University

${ }^{4}$ Chitkara University Research \& Innovation Network, Chitkara University, Punjab, INDIA
} 
these lines require careful resource organization. The sensor centers are thickly sent either inside the sink or close it and have confined power, computational breaking point and memory. Sensor center points are to a great degree slanted to frustrations. Sensor centers won't not have overall unmistakable evidence (ID) because of the considerable measure of overhead. Sensor center points are thickly passed on in broad numbers. Thusly, the significant target of a WSN is to make information from distinguished data by singular sensor mode by deferring the life time of WSN The compelled drive of sensor center points arranges the arrangement of essentialness capable correspondence tradition. The movement in remote sensor frameworks causes overabundance and impacts the imperativeness and transmission limit utilization. Examiners have invented various traditions for correspondence, and security in remote frameworks life structure based frameworks, exceptionally designated frameworks, flexible frameworks, et cetera [14].

Power and steering mindfulness is united in tradition stack and join the information with frameworks organization traditions, and transmit the imperativeness competently in the midst of the remote medium. Many layers are fused into tradition stack, for instance, data associate, physical layer, transport, control organization sheet and errand organization plane. Diverse kind of use programming can be made that depends on upon the recognizing errand and can be used by application layer. Transport layer manage the surge of the information if it can be required by the sensor frameworks application layer. Sort out layer can be disturbed of controlling the information gave by the vehicle layer. Mac traditions should be control careful and fit to reduce crash with neighbors transmit if the surroundings is uproarious. Physical layer check the necessities of basic yet fiery artificiality, correspondence and getting techniques. Plus, the control, quality and errand organization planes watch the power, development and task circulation between the sensor centers. These planes enable the sensor center points to interrelate the disclosure development and lesser the wide power utilize [15]. Most of the computational techniques regular handling oversees rely upon exceedingly streamlined variations of the instruments and methods present in the contrasting ordinary miracles. The reasons behind such changes and consultations are perplexing. As an issue of first significance, most separations are vital to make the count with a broad number of components tractable. In like manner, it can be gainful to feature the irrelevant segments critical to enable some particular parts of a structure to be reproduced and to observe some emanant properties. In this paper normal processing is utilized to course the information from source to the BS in a WSN. As the proposed system is impelled by the nature so it plays out the endeavor in a streamlined and powerful way [16].

\section{RELATED WORK}

This area covers the different calculations intended for the remote sensor organize. Distinctive planning calculation for the WSN have been secured by the creator of [17] while the directing conduct has been portrayed here. Majumder et.al. [18] proposes another power-mindful, adaptable, different leveled and chain based tradition - CCPAR (Clustered Chain based Power Aware Routing) that uses the incidental assignments of the group rush toward different center points in light of the most significant residual battery constrain as to ensuring the even dispersal of compel by each one of the center points. Transmission from a lone gathering scramble toward the base station in each round and the transport of the data add up to workload among each one of the center points, save the group heads from early weakness. The usage of data amassing moreover decreases the measure of information to be transmitted to the base station. By tying the center points in each gathering and using an alternate chain for the pack heads, CCPAR offers the upside of little transmit partitions for a substantial bit of the centers and accordingly encourages them to be operational for a more drawn out time span by observing their limited essentialness. This tradition has been shown better than anything the other standard traditions LEACH [19], PEGASIS [20], TEEN [21], APTEEN [22]. Chih-Chung Lai et. al. [23] proposes a hereditary figuring to handle the DSC issue. The Disjoint Set Covers (DSC) finds the most outrageous number of sensor spreads can be settled by methods for change as more sensor spreads can be found, to draw out framework lifetime. Ying Lin et. al. [24] proposes an Ant Colony Optimization-based Approach for Maximizing the Number of Connected Covers (ACO-MNCC) to support the lifetime of heterogeneous WSNs. The procedure relies upon finding the most extraordinary number of disjoint related spreads that satisfy both distinguishing extension and framework organize. An improvement diagram is made with each vertex meaning the errand of a device in a subset. In perspective of pheromone and heuristic information, the ants search for a perfect path on the improvement graph to help the amount of related spreads. The pheromone fills in as a likeness for the interest experiences in building related spreads. The heuristic information is used to reflect the appeal of contraption assignments. An area look system is planned to furthermore improve the chase capability. Akira Mutazono et. al. [25] proposed approach has been associated with a variety of heterogeneous WSNs to its significance. propose a self-dealing with planning design charged by Japanese tree frog calling conduct for imperativeness capable data transmission in remote sensor frameworks. The frogs are known to make approaches the other hand in light of their neighbors keeping the ultimate objective to raise the probability of mating. This lead can be associated with organize control that recognizes crash free transmission getting ready for remote correspondence. These frogs in like manner demonstrate a sort of lead known as satellite direct, where a frog stops calling once it recognizes the calls of other neighboring frogs. This direct can be associated in the diagram of an essentialness capable rest control framework that gives flexible operation periods. This paper plans a Moth-Dolphin based directing calculation for WSN examined in additionally segments.

\section{MOTH -FLAME OPTIMIZATION (MFO) ALGORITHM}

Moth is a type of butterfly and it uses the moon radiance (light) to route towards the moon. The routing of the moth comprises of straight as well as the spiral motion depending upon whether the moon(destination) is far or near from the current position 
of moth. This phenomenon of routing of the moth towards the moon can be used to in sensor network to transfer the data from source to the destination in an optimized manner [1]. This section describes the moth-flame optimization algorithm as follow In the MFOA, $\mathrm{n}$ moths are travelling towards the moon in d dimensions then the position matrix can be given shown as eq.(1) $P_{m}=\left[\begin{array}{ccc}P_{1,1} & \cdots & P_{1, d} \\ \vdots & \ddots & \vdots \\ P_{n, 1} & \cdots & P_{n, d}\end{array}\right]$

The evaluation of the position of the moth can be done by using the objective (fitness) function and corresponding matrix is given in eq. (2).

$F_{v_{-} m}=\left[\begin{array}{c}F_{v_{-} m 1} \\ \vdots \\ F_{v_{-} m n}\end{array}\right]$

The best position in the matrix of equation (1) can be found by analyzing the corresponding values in eq. (2) (best solution can be minimum or maximum value depending upon the problem). The best position vector is the form of a matrix (known as flame matrix) displayed in eq. (3).

$P_{f}=\left[\begin{array}{ccc}P_{-} f_{1,1} & \cdots & P_{-} f_{1, d} \\ \vdots & \ddots & \vdots \\ P_{-} f_{n, 1} & \cdots & P_{-} f_{n, d}\end{array}\right]$

The corresponding values of the objective function in shown in the equation (4)

$F_{v_{-} f}=\left[\begin{array}{c}F_{v_{-} f 1} \\ \vdots \\ F_{v_{-} f n}\end{array}\right]$

When the moth reaches nearer to the moon then the moth starts to follow the spiral motion which can be specified by using eq.(5) as shown:

$P_{m_{i}, f_{j}}=F_{v_{-} f j}+\left|F_{v_{-} f j}-F_{v_{-} m i}\right| * \cos (2 \pi r) * e^{b r}$

The $P_{m i, f_{j}}$ denotes the updated position using the spiral motion while the $\mathrm{b}$ is a constant to define shape of the motion. The $\mathrm{r}$ is used to define the moth and flame relationship if the value of $r$ is 1 then the moth follows the flame, resulting the best possible position near the flame is selected otherwise the moth selects the best far position from the flame. This updated position is used to move towards the optimization. The number of flames is decreasing with the increase in iteration which can be represented by eq. (6)

Counting $($ flames $)=\operatorname{ceil}\left(n-\right.$ iteration $\left._{n o} * \frac{n-1}{\text { Total }_{\text {iteration }}}\right)$

Where the total number of iterations and the current iteration number is given by the Total $_{\text {iteration }}$ and iteration $_{n o}$ respectively [1]. This process is used attain the global optima. The detail of dolphin has been described in next section

\section{MOTH FLAME BASED ROUTING ALGORITHM IN WIRELESS SENSOR NETWORK (MFRA)}

The Moth-flame optimization algorithm (MFOA) [1] depicts the nature of butterfly to fly towards moon. This algorithm has been proved better than various existing state of art techniques [1]. The MFOA can be applied for the routing in the wireless sensor network (WSN). The WSN consists of $\mathrm{N}$ sensor nodes with sensing as well as communication features to send the sensed data. These nodes also forwards the data received from previous node towards the base station. Here, each data packet is considered as the moth which moves towards the base station, taken as moon. The data packet follows the straight motion when the packet is far away from the base station; otherwise the spiral motion is followed as in the case of MFOA. The process of Moth-Flame based routing in WSN can be elaborated as follow:

In the MFRA, $\mathrm{n}$ nodes (moth) to transfer data from source towards the base station BS (moon) in 2 dimensions(x,y coordinates) then corresponding position matrix can be given shown as eq. 7.

$P_{d}=\left[\begin{array}{cc}P_{1,1} & P_{1,2} \\ \vdots & \vdots \\ P_{n, 1} & P_{n, 2}\end{array}\right]$

The distance of the node from the BS is used to calculate the fitness value so,

$F_{d \_m}=\left[\begin{array}{c}F_{d \_m 1} \\ \vdots \\ F_{d \_m n}\end{array}\right]$

Where,

$F_{d_{m i}}=\sqrt{\left(P_{i, 1}-B S_{1}\right)^{2}+\left(P_{i, 2}-B S_{2}\right)^{2}}$ 
Here, BS1 and BS2 is the $\mathrm{x}$ and $\mathrm{y}$ coordinates of base station respectively. The best position in the matrix of equation (7) is the entry with minimum fitness value in eq. (8). The best position vector is marked as flame which is given by eq. (10)

$P_{f}=\left[\begin{array}{cc}P_{-} f_{1,1} & P_{-} f_{1,2} \\ \vdots & \vdots \\ P_{-} f_{n, 1} & P_{-} f_{n, 2}\end{array}\right]$

The corresponding values of the objective function in shown in the equation (11)

$F_{d_{-} f}=\left[\begin{array}{c}F_{d_{-} f 1} \\ \vdots \\ F_{d_{-} f n}\end{array}\right]$

Now, compare the values of eq.(11) with the corresponding entry of the eq. (8) and perform the eq. (12)

if $\left(F_{d_{f i}}>F_{d_{-} m i}\right)\left\{\right.$ Replace $P_{f i}$ vector in $P_{f}$ by $P_{i}$ and $\left.\left.F_{d_{f i}}=F_{d_{-} m i}\right)\right\}$

If the position is updated only if corresponding fitness value is better than the existing one. When the packet reaches nearer to the BS then it starts to follow the spiral motion which can be specified by using eq.(13) as shown:

$P_{m_{i}, f_{j}}=F_{d_{-} f j}+\left|F_{d_{-} f j}-F_{d_{-} m i}\right| * \cos (2 \pi r) * e^{b r}$

The $P_{m i, f_{j}}$ denotes the updated position using the spiral motion while the $\mathrm{b}$ is a constant to define shape of the motion. The $\mathrm{r}$ is used to define the moth and flame relationship if the value of $r$ is 1 then the moth follows the flame, resulting the best possible position near the flame is selected otherwise the moth selects the best far position from the flame. This updated position is used to move towards the optimization. The number of flames is decreasing with the increase in iteration which can be represented by eq. (14)

Counting $($ flames $)=\operatorname{ceil}\left(n-\right.$ iteration $\left._{n o} * \frac{n-1}{\text { Total }_{\text {iteration }}}\right)$

Where the total number of iterations and the current iteration number is given by the Total $_{\text {iteration }}$ and iteration $_{\text {no }}$ respectively. In the MFOA the moth are moving and changing their positions while in the MFRA the position of nodes is fix and the data packet selects a node with the location nearest to the updated position. It means MFRA gives the updated position of the data packet on the basis of the position of the node. The step by step description of the Moth Flame based Routing Algorithm in Wireless Sensor Network (MFRA) is defined below:

4.1 MFRA(n, P[][], data, $S, B S)$

The MFRA is implemented on a network with $\mathrm{n}$ sensor nodes with corresponding position vector is given by $\mathrm{P}_{\mathrm{d}}[][]$. The node $\mathrm{S}$ wants to route the "data" to the base station (BS).

1. Initiate Network with n nodes:

For $\mathrm{i}=1: \mathrm{n}$

Calculate fitness value

End

$$
F_{d_{m i}}=\sqrt{\left(P_{i, 1}-B S_{1}\right)^{2}+\left(P_{i, 2}-B S_{2}\right)^{2}}
$$

2 .

3. $\quad$ Current $_{\text {node }}=\mathrm{S}$

4. While Current node $\neq B S$

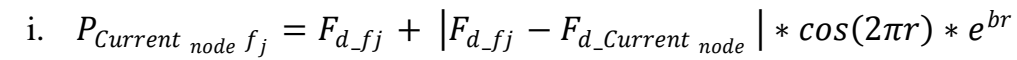

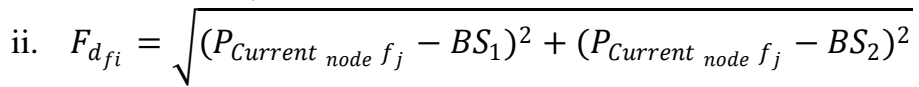

iii. if $\left(F_{d_{f i}}>F_{d_{-} m i}\right)\left\{\right.$ Replace $P_{f i}$ vector in $P_{f}$ by $P_{i}$ and $\left.F_{d_{f i}}=F_{d_{-} m i}\right\}$

iv. Select Next Node in The route

v. $\mathrm{F}_{\mathrm{dN}}=$ QuickSort $\left(F_{d_{f i}}, F_{d \text { current }}{ }_{\text {node }}\right)$

vi. End if

vii. Update $b$ and $t$

viii. Update Current ${ }_{\text {node }}$

End while

5. Exit 
The above algorithm is used route the data from source (S) to the base station (BS). The algorithm can be implemented for optimized routing in wireless sensor network.

\section{CONCLUSION}

This paper discusses the natural computing techniques along with their application in the wireless sensor network. The paperalso describes the moth flame optimization algorithm which describes the behavior of moth to move towards the flame. Then the behavior of moth is used for routing the data towards destination in the wireless sensor network. This algorithm uses the moth behavior along with the distance of neighbor from current node to route the data in multi hop fashion towards the destination. In future this algorithm can be implemented for optimized routing in wireless sensor network.

\section{REFERENCES}

[1] Mirjalili S. Moth-flame optimization algorithm: A novel nature-inspired heuristic paradigm. Knowledge-Based Systems. 2015 Nov 30;89:228-49.

[2] [2] de Castro LN. Fundamentals of natural computing: an overview. Physics of Life Reviews. 2007 Mar 31;4(1):1-36

[3] [3] D. E. Goldberg, \& J. H. Holland, Genetic algorithms and machine learning, Machine learning 3(2) (1988) 95-99.

[4] [4] R. C. Eberhart, \& J. Kennedy, A new optimizer using particle swarm theory, In Proceedings of the sixth international symposium on micro machine and human science 1 (1995) 39-43.

[5] [5] R. Storn, \& K. Price, Differential evolution-a simple and efficient heuristic for global optimization over continuous spaces, Journal of global optimization 11(4) (1997) 341-359.

[6] [6] E. Rashedi, H. Nezamabadi-Pour, \& S. Saryazdi, GSA: A gravitational search algorithm. Information Sciences, 179(13) (2009) 2232-2248.

[7] [7] S. Mirjalili, S. M. Mirjalili, \& A. Lewis, Grey wolf optimizer, Advances in Engineering Software 69 (2014) 46-61

[8] [8] Deb K, Pratap A, Agarwal S, Meyarivan TA. A fast and elitist multiobjective genetic algorithm: NSGA-II. Evolutionary Computation, IEEE Transactions on. 2002 Apr;6(2):182-97.

[9] [9] Nguyen HB, Xue B, Liu I, Andreae P, Zhang M. New mechanism for archive maintenance in PSO-based multi-objective feature selection. Soft Computing. 2016:1-20.

[10] [10] Vieira SM, Mendonça LF, Farinha GJ, Sousa JM. Modified binary PSO for feature selection using SVM applied to mortality prediction of septic patients. Applied Soft Computing. 2013 Aug 31;13(8):3494-504.

[11] Lee JW, Choi BS, Lee JJ. Energy-efficient coverage of wireless sensor networks using ant colony optimization with three types of pheromones. IEEE Transactions on Industrial Informatics. 2011 Aug;7(3):419-27.

[12] J.-Y. Heo, J.-M. Hong, and Y.-K. Cho, "EARQ: Energy aware routing for real-time and reliable communication in wireless industrial sensor networks," IEEE Trans. Ind. Informat., vol. 5, no. 1, pp. 3-11, Feb. 2009.

[13] Dongfeng Xie et al., "A chain-based data gathering protocol under compressive sensing framework for wireless sensor networks", 2013 International Conference on Computational and Information Sciences

[14] Sridhar Sharma, "A Survey of Hierarchical Routing Protocols in Wireless Sensor Networks", Volume 3, Issue 12, December 2013

[15] Neha Singh et al., "Wireless Sensor Networks: Architecture, Protocols, Simulator Tool”, Volume 2, Issue 5, May 2012

[16] de Castro LN. Fundamentals of natural computing: an overview. Physics of Life Reviews. 2007 Mar 31;4(1):1-36.

[17] Musilek P, Krömer P, Bartoň T. Review of nature-inspired methods for wake-up scheduling in wireless sensor networks. Swarm and Evolutionary Computation. 2015 Dec 31;25:100-18.

[18] Majumder K, Ray S, Sarkar SK. A novel energy efficient chain based hierarchical routing protocol for wireless sensor networks. In Emerging Trends in Robotics and Communication Technologies (INTERACT), 2010 International Conference on 2010 Dec 3 (pp. 339-344). IEEE.

[19] Xiangning F, Yulin S. Improvement on LEACH protocol of wireless sensor network. InSensor Technologies and Applications, 2007. SensorComm 2007. International Conference on 2007 Oct 14 (pp. 260-264). IEEE.

[20] Lindsey S, Raghavendra CS. PEGASIS: Power-efficient gathering in sensor information systems. InAerospace conference proceedings, 2002. IEEE 2002 (Vol. 3, pp. 3-3). IEEE.

[21] Manjeshwar A, Agrawal DP. TEEN: ARouting Protocol for Enhanced Efficiency in Wireless Sensor Networks. Inipdps 2001 Apr 23 (Vol. 1, p. 189).

[22] Manjeshwar A, Agrawal DP. APTEEN: A Hybrid Protocol for Efficient Routing and Comprehensive Information Retrieval in Wireless Sensor Networks. InIpdps 2002 Apr 15 (Vol. 2, p. 48).

[23] Lai CC, Ting CK, Ko RS. An effective genetic algorithm to improve wireless sensor network lifetime for large-scale surveillance applications. InEvolutionary Computation, 2007. CEC 2007. IEEE Congress on 2007 Sep 25 (pp. 3531-3538). IEEE.

[24] Lin Y, Zhang J, Chung HS, Ip WH, Li Y, Shi YH. An ant colony optimization approach for maximizing the lifetime of heterogeneous wireless sensor networks. IEEE Transactions on Systems, Man, and Cybernetics, Part C (Applications and Reviews). 2012 May;42(3):408-20.

[25] Mutazono A, Sugano M, Murata M. Energy efficient self-organizing control for wireless sensor networks inspired by calling behavior of frogs. Computer Communications. 2012 Mar 15;35(6):661-9. 